\title{
Improvement of Proton Beam Quality from the High-intensity Short Pulse Laser Interaction with a Micro-structured Target
}

\author{
Ju Tae Seo', Seung Hoon Yoo, ${ }^{1,2}$ Ki Hong Pae ${ }^{1,3}$, and Sang June Hahn ${ }^{1 *}$ \\ ${ }^{1}$ Department of Physics, Chung-Ang University, Seoul 156-756, Korea \\ ${ }^{2}$ Advanced X-ray Medical System Research Group, Korea Electrotechnology Research Institute, \\ Ansan 426-170, Korea \\ ${ }^{3}$ Advanced Photonics Research Institute, Gwangju Institute of Science and Technology, \\ Gwangju 500-712, Korea
}

(Received November 8, 2008 : revised February 2, 2009 : accepted February 2, 2009)

\begin{abstract}
Target design study to improve the quality of an accelerated proton beam from the interaction of a high-intensity short pulse laser with an overdense plasma slab has been accomplished by using a two-dimensional, fully electromagnetic and relativistic particle-in-cell (PIC) simulation. The target consists of a thin core part and a thick peripheral part of equivalent plasma densities, while the ratio of the radius of the core part to the laser spot size, and the position of the peripheral part relative to the fixed core part were varied. The positive effects of this core-peripheral target structure could be expected from the knowledge of the typical target normal sheath acceleration (TNSA) mechanism in a laser-plasma interaction, and were apparently evidenced from the comparison with the case of a conventional simple planar target and the case of the transversal size reduction of the simple planar target. Improvements of the beam qualities including the collimation, the forward directionality, and the beam divergence were verified by detailed analysis of relativistic momentum, angular directionality, and the spatial density map of the accelerated protons.
\end{abstract}

\footnotetext{
Keywords: Laser-plasma interaction, Micro-structured target, PIC simulation, Proton beam generation

OCIS codes : (140.0140) Lasers and laser optics; (320.0320) Ultrafast optics; (350.0350) Other areas of optics
}

\section{INTRODUCTION}

During the last decades, rapid developments in laser technology including chirped pulse amplification (CPA) have enabled high power lasers [1] to produce multiterawatt femtosecond pulses that allow the examination of the fundamental physics of particles (-including protons) with acceleration to multi-MeV energies. Since its early stage of theoretical study $[2,3]$ and experimental verification [4], generation of energetic proton from high -intensity laser irradiated thin foil targets has received much scientific attention during the past decade, and has been considered as a key issue for many possible applications in various fields of physics, including fast ignition of inertial confinement fusion (ICF) [5], X-ray

\footnotetext{
*Corresponding author: sjhahn@cau.ac.kr
}

sources [6], nuclear physics [7], astrophysics [8], and radiation therapy [9].

Generation of a collimated, energetic proton (ion) beam by interacting a short duration high-intensity laser pulse with a solid foil target has been observed in numerous experiments and in numerical simulations. Based on the several controversial studies [10-14], it has been well-known that the energetic protons can originate from both sides of a target (the front surface and the rear surface, with respect to the propagation direction of the incident laser pulse) depending on the laser parameters such as the intensity and the duration, and the target parameters such as the thickness, the density, and the geometric profile [15-23].

The quality of the accelerated ion beam can be described by several aspects, such as beam emittance and energy spread. According to a series of reports [10-14], 
the maximum achievable energy of the accelerated ions is essentially predetermined by the laser pulse intensity, regardless of the acceleration mechanism. It should be more meaningful to discuss the improvement of the beam quality in the moderate range of the laser intensity $\left(I_{L}\right.$ $<$ few $10^{20} \mathrm{~W} / \mathrm{cm}^{2}$ ). For these moderate intensities, the recent relevant studies [23-25] showed that the fundamental properties of the beam quality are mainly determined by the target parameters rather than the laser parameters, as mentioned previously, the thickness, the density, and the geometric profile of the target rather than the intensity and the duration of the irradiated laser pulse. Furthermore, as described by H. Schwoerer et al. [24], the transversal size reduction of the target is desirable to avoid the transversal inhomogeneity of a laser pulse for the ion acceleration. It is essentially due in the nature of a laser pulse of which the spot size $\left(\sigma_{L}\right)$ does not mean the actual boundary between the bright part and the dark part on the target surface, similarly to the fact that in general, a main pulse accompanies the weaker but longer prepulse which always precedes the main pulse itself. Considering the fundamental mechanisms of ion acceleration, it can be expected that the characteristics of the ion acceleration can be largely affected by the variety of the microscopic transversal structure of the target, as well as the longitudinal structure (e.g., thickness of a simple planar target).

In the previous work, we showed that the quality of the accelerated proton beam can be enhanced by using a transversally structured target which consists of a dense core part and relatively tenuous peripheral part, with the same thickness [26]. In the result, as expected, existence of the peripheral part positively contributed for the TNSA mechanism to the protons in the core part. In this work, our main interest is hence to investigate the effect of the enriched peripheral part (i.e., enriched peripheral electrons) to the acceleration of the core part. To avoid difficulties in experimental verification, we again assume a laboratory class laser system $\left(I_{L} \approx 10^{20} \mathrm{~W} / \mathrm{cm}^{2}\right)$, and furthermore, we let the target plasma densities of the two parts to be equal. Consequently the difference of the two parts of the target comes from the different thicknesses, rather than the different plasma densities.

The remain parts of this paper are organized as follows. In the next section, brief scheme of a fully relativistic 2D3V (x,y, $v_{x}, v_{y}$, and $\left.v_{z}\right)$ particle-in-cell (PIC) simulation will be introduced. The next section has three subsections showing the fundamental characteristics of proton acceleration from a simple planar target, the effect of transversal size reduction of the simple planer target and its limitation, and the improvement of beam quality from the proposed microstructured target, respectively. The conclusion and the suggestion for further study will follow.

\section{SIMULATION SCHEME}

In the simulation work, we consider a linearly polarized laser pulse with a wavelength $\lambda_{L}=1 \mu \mathrm{m}$, normally incident onto a target. The pulse duration is $\tau_{L}=30$ $\mathrm{fs}$, and the full-width at half-maximum (FWHM) of the laser pulse is $1.18 c \tau_{L}$ where $\mathrm{c}$ is speed of lights in a vacuum. A laser pulse with vacuum electric field amplitude $E_{L}$ and with a spot size $\sigma_{L}=5 \mu \mathrm{m}$ is assumed to be injected at the left boundary of the simulation box and to propagate in the $\mathrm{x}$ direction of an $(x, y)$ simulation plane. The normalized amplitude of the laser vector potential, $a=e E_{L} / m c \omega_{L}$, was fixed as 10.0, corresponding to laser intensities $I_{L}$ of $1.38 \times 10^{20} \mathrm{~W} / \mathrm{cm}^{2}$.

The target is located at $x_{0}=200 \mu \mathrm{m}$ from the left boundary of the simulation box, assumed to be a fully ionized cold plasma slab with the typically-chosen full thicknesses of 1 and $3 \lambda_{L}$, and is composed of protons and electrons with equal densities, as shown in Fig. 1. In the micro-structured target, the central core part has a comparable size of radius $\left(r_{c}\right)$ with the laser spot size $\left(\sigma_{L}\right)$. The plasma densities of the two parts of target - core $\left(n_{c}\right)$ and peripheral $\left(n_{p}\right)$ part - are set commonly as $16 n_{c r}$. For the detailed study, location of the peripheral part of target varied forwards and backwards while the core part is fixed at $x=200 \lambda_{L}$. Actually, this structure is one of the simplest modifications of target structure since it can be easily achieved by digging microscopic depressions on the surfaces (front or rear side) of a typical planar target of few- $\mu$ m's thickness. Here $n_{c r}=$ $1.11 \times 10^{21} \mathrm{~cm}^{-3}$, is the critical density of the laser pulse. Since collisionless skin depth, $\delta=0.042 \mu \mathrm{m}$ is smaller than any size of the microscopic structures considered in this work, the whole target is not transparent to the

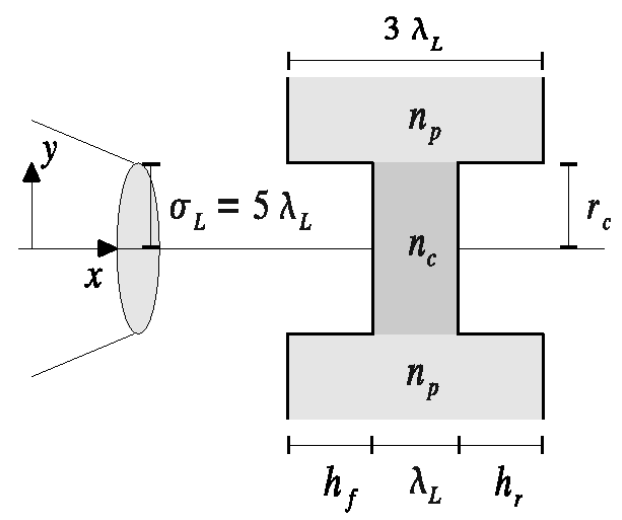

FIG. 1. Schematic concept of short-pulse laser interactions with a core-peripheral structured plasma slab target. The two parts have an identical plasma density $\left(n_{c}=n_{p}=\right.$ $\left.16 n_{c r}\right)$, while thicknesses are different. The incident laser pulse of 30 fs duration and of $1 \mu \mathrm{m}$ wavelength irradiates on the core part normally with a spot size of $5 \lambda_{L}$. The laser intensity at the spot position was also fixed as 1.38 $\times 10^{20} \mathrm{~W} / \mathrm{cm}^{2}$ in this study. 
laser pulse.

The total simulation domain was $256 \lambda_{L} \times 128 \lambda_{L}$ and the total simulation time was over 1 ps. The computational resolutions for space and time are approximately $\lambda_{L} / 10$ and $\tau_{L} / 100$, respectively. Here, the maximum electron density of the plasma slab and the size of our simulation box are limited by computer capabilities.

We have performed 2D PIC simulation by using XOOPIC code developed in Plasma Theory and Simulation Group, University of California at Berkeley [27]. The effect of the preplasmas, which may be induced by the prepulse of an intense laser, was not considered in this work, by assuming the use of the laser pulse with a sufficiently high contrast ratio in the propagation direction.

\section{SIMULATION RESULTS}

In an interaction of a short laser pulse with a simple, thin planar plasma slab target, the most energetic ions are found to come from the front, the bulk, or the back of the target depending on the laser intensity and pulse duration. When the laser pulse hits the target, electrons are mostly heated by the $\mathbf{j} \times \mathbf{B}$ mechanism and pushed inwards by the laser ponderomotive force. A charge separation is induced at the front surface. The resulting electrostatic field accelerates the target surface forward by hole-boring. At the back surface, the electron bunches produced by the laser and transported through the target create a strong electrostatic field that drives surface ion acceleration. This electrostatic field acceleration (plasma expansion) model well explains both the forward ion acceleration at the rear surface (FIAR) and the backward ion acceleration (BIA) at the front surface. As the laser intensity becomes higher, the ion wave, launched into the front side of target, becomes fast enough so that it can accelerate the ions initially at rest in its path. These so-called collisionless shocks are often encountered during the interaction of a laser pulse with an overdense plasma. This shock acceleration model contributes only to the forward ion acceleration at the front surface (FIAF) [14].

In the previous work [26], the target consists of a dense core part and a relatively tenuous peripheral part with the same thickness. While in the present work, the target consists of the core and the peripheral parts with different thicknesses. Since our focus in this study is to estimate the contribution of the peripheral part for the proton acceleration, we considered when the peripheral part is totally absent so that the transversal size of a typical planar target was simply reduced to the laser spot size. The effects of this transversal size reduction were considered for two different cases of target thickness, $L=$ $3 \lambda_{L}$ and $\lambda_{L}$, respectively. However, the protons from the transversally size-reduced target have an undesirably broad distribution of angular directionality compared to the selected near-axis protons from the transversely total-expanded target (i.e., the simple planar target in the previous discussion). Furthermore, as there are fewer electrons which are leading the TNSA for the target protons, the reachable relativistic momenta along the $x$ direction $\left(\gamma v_{x}\right)$ of the protons were not improved though there was some increment of total $\gamma v$ to the diffusive direction. Here, $\gamma$ is the relativistic factor defined by $\left(1-\mathrm{v}^{2} / \mathrm{c}^{2}\right)^{-1 / 2}$. The reason for the broad distribution of angular directionality of the accelerated protons seems to naturally come from the acceleration mechanism itself. For a simple planar target, there is a bunch of protons which is originated from the peripheral part of the target, forming the conic structure. The conic structure of protons forms the inward electric field from the peripheral side to the core side (i.e., axis of the acceleration), hence the angular distribution of the near-axis protons can be somewhat enhanced. For the transversely size-reduced planar target, in contrast, as there is no protons in the peripheral part, there is no factor for the core protons to have the better angular distribution though they could be accelerated definitely forward at the initial stage. And then, since they have purely positive charges, they should rapidly diffuse to the transverse direction as they leave the target surface. In physical respects, these differences of proton acceleration characteristics between the simple planar target and the size-reduced planar target could be expected from the different electric field patterns formed by the electrons with different population and distribution, especially in this TNSA-dominant regime. As a conclusion, the transversal reduction of the simple planar target does not give advantageous features to the important properties (angular directionality and spatial structure) of the energetic proton beam, compared to the case of the simple planar target

In the proton acceleration by the laser-plasma interaction, the resultant characteristics of the accelerated proton beams are known to be strongly dependent on the target conditions such as density, thickness, and target geometry. The angular directionality distribution of the proton beam is defined by the three-dimensional structures of the accelerating fields both at the front and the rear side of the target. In the dominant FIAR acceleration, the angular distribution resembles the 3D structure of the sheath electrostatic field at the rear side of the target. The typical ejection pattern of the proton beams is an annular conic shape. In general, the predominant FIAR has a good collimation and a low emittance and the FIAF protons are divergent and limited to low energies. Recently, for achieving betterquality and more energetic proton beams, the modified targets based on the thin foil target have been considered [28-30]. In this work, based on the knowledge for the ion acceleration with a simple plasma slab target 


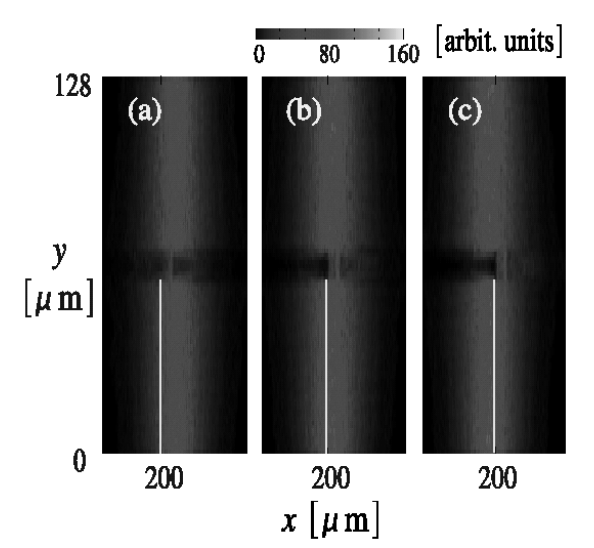

FIG. 2. (color online) Target density maps after the laser irradiation on the front surface of the core part: the cases are (a) $h_{f}=2 \lambda_{L}$, (b) $h_{f}=h_{r}=1 \lambda_{L}$ (centered case), and (c) $h_{f}=0$, respectively, and the core radius $\left(r_{c}\right)$ is equal to the laser spot size $\left(\sigma_{L}\right)$. The laser intensity and the core target location were fixed as $a=10\left(I_{L}=1.38\right.$ $\times 10^{20} \mathrm{~W} / \mathrm{cm}^{2}$ ), and $x=200 \lambda_{L}$ (indicated by yellow lines), respectively.

from the previous works, we have proposed a microstructured target as shown in Fig. 1. The typical planar target is modified both in the transverse and longitudinal dimensions, in which the typical thin central core part has been encircled by a thicker peripheral part. Since the plasma densities in the two parts are identical, the peripheral part can be described as spatially enriched compared to the simple planar target. Here the target modification is controlled by varying the core radius $\left(r_{c}\right)$ and the distance of the peripheral surface from the core surface $\left(h_{f}\right.$ or $\left.h_{r}\right)$ as illustrated in Fig. 1. It should be noted here that the thicknesses of the core part and the peripheral part were fixed as $\lambda_{L}$ and $3 \lambda_{L}$, respectively.

The relative locations of the peripheral parts to the fixed $(x=200 \mu \mathrm{m})$ central core part are shown in Figs. 2(a), (b), and (c). By these representative cases, the following physical pictures are expected. First, when the core part is located at the front surface of the peripheral part $\left(h_{f}=0\right.$ in Fig. 1), as the laser pulse irradiates directly the surface of the core target, the core protons meet both the forward and the backward forces similarly to a simple planar target without any modifications. But it can be also expected that the ponderomotive forces to the both sides are not balanced by the broken symmetry of the target structure. Since the core part is located at the front surface of the peripheral part, the electric field from the forwardly ( $+x$ direction at the rear surface of the peripheral part) expanding peripheral electrons is shielded out by the protons in the peripheral part, hence becomes weaker than the electric field from the backwardly $(-x$ direction at the front surface of the peripheral part) expanding

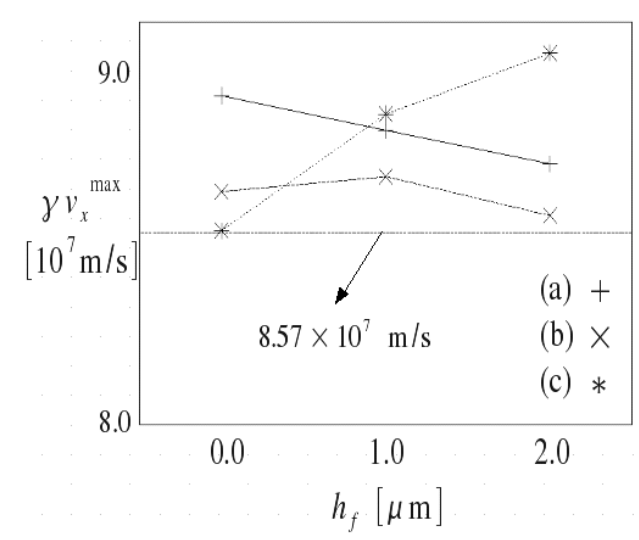

FIG. 3. Comparison of the maximum relativistic momenta of the energetic core protons from the structured target and a simple planar target of core thickness. The radius of core part varied as (a) $r_{c}=0.4 \sigma_{L}$, (b) $r_{c}=\sigma_{L}$, and (c) $r_{c}=2 \sigma_{L}$, respectively, where $\sigma_{L}=5 \lambda_{L}$ is fixed. The horizontal line at $\gamma v_{x}^{\max }=8.57 \times 10^{7} \mathrm{~m} / \mathrm{s}$ indicates the result for a simple planar target of core thickness $\left(1 \lambda_{L}\right)$.

electrons. Even worse, as the accelerated protons from the core part should go through the channel in the peripheral target, the beam quality of the core protons at the rear surface of the target (i.e., at the rear surface of the peripheral part) may not be good enough. Of course, however, it can be better than the simple planar target of core thickness $\left(\lambda_{L}\right)$ because of the totally enriched population of the electrons.

The second possibility of the core target location is the center of the peripheral part $\left(h_{f}=h_{r}=\lambda_{L}\right.$, centered case). In this centered case, as protons from the peripheral part partially shield the electric fields out from both directions, forward and backward, the ponderomotive force induced by the peripheral electrons can exert on the core target symmetrically but not with the full strength. What should be taken into account here is that the core protons inside the tunnel feel not only the electric field from the peripheral electrons but also the electric field from the core electrons, since a majority of the incident laser pulse can directly irradiate the core target if the core radius is not too small. The net source of the TNSA for the core target hence comes from both of the two electric fields.

Thirdly, and lastly, when the core target is located at the rear surface of the peripheral part $\left(h_{r}=0\right)$, the core protons will feel mainly the electric field from the forwardly expanding electrons, in contrast to the case when the core target is located at the front surface of the peripheral part. However, the laser pulse should propagate through the long channel inside the peripheral part to reach the surface of the core target, and the effect of direct irradiation can be somewhat decreased for the core target.

With these expectations, we performed parametric 


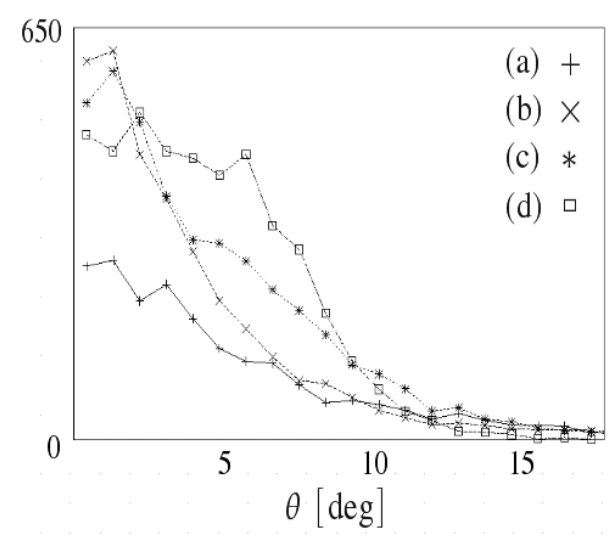

FIG. 4. Comparison of the angular directionality of the energetic core protons from the structured target of $r_{c}=$ $\sigma_{L}$ (case (b) in Fig. 5) and a simple planar target of core thickness. The varying distances between core and peripheral parts are (a) $h_{f}=0$, (b) $h_{f}=h_{r}=\lambda_{L}$ (centered case), and (c) $h_{f}=2 \lambda_{L}$, respectively (Fig. 4 (a)-(c)). (d) Result for a simple planar target of core thickness $\left(1 \lambda_{L}\right)$.

studies along the varying $r_{c}$, and the most evident and remarkable results come from the case $r_{c}=\sigma_{L}$, when the core radius is equal to the laser spot size. Figure 3 shows the compared maximum proton energies at near to $1 \mathrm{ps}$ after the laser irradiation on the core target surface. The result from the simple planar target of core thickness (i.e., without the enriched peripheral part) was also given in the figure for comparisons. The enrichment of the peripheral part commonly brought the enhancement of the maximum energy of the accelerated protons, as we expected previously. One fact to be noted here is, however, when the core radius is small, the most energetic particle occasionally originates in the peripheral part rather than the core part. For such a tiny -scaled core structure, it is more desirable to treat the target as a simple planar target of peripheral thickness rather than the core.

The comparison of the angular directionality distributions of the energetic protons from the core target is shown in Fig. 4. The result from the simple planar target of core thickness is also shown. In contrast to the maximum proton energy, not all cases show the significant enhancement from the simple planar target. However, for the centered case, when the core target is centered in the tunnel of the peripheral part, it is evident that the angular directionality distribution has been remarkably improved compared to the other cases, including the simple planar target. In this centered case, over $80 \%$ of the energetic protons are traveling in the angular ranges from zero to $5^{\circ}$, with respect to the laser propagation. The peak position near zero, in the distribution, also strongly implies that the energetic proton beam quality can be greatly improved by use of a properly, but relatively simply, structured target.

\section{CONCLUSION}

In this work, we have studied the effects of the modifications to the microscopic structures of a target for the energetic proton generation from a high-power laser-plasma interaction. Based on the fundamental mechanisms of laser-plasma interactions and the resultant characteristics of the high-energy protons generated, we showed that the mere size reduction of a slab target does not give improvements to the beam quality of the accelerated protons. The reason for this can be summarized as the lack of the TNSA-leading electrons and, the diffusive motion of the target protons. We also investigated a simple but effective structure of the target which improved the important qualities of the energetic proton beam, especially the relativistic momentum and the angular directionality. The most desirable result came from use of the centered core-peripheral target structure, which is easily constructible by digging a simple planar target in certain depth on both surfaces of the target.

\section{ACKNOWLEDGEMENT}

This work was supported by the Chung-Ang University Research Grant in 2007.

\section{REFERENCES}

1. Y. Izawa, N. Miyanaga, J. Kawanaka, and K. Yamakawa, "High power lasers and their new applications", J. Opt. Soc. Korea 12, 178-185 (2008).

2. S. C. Wilks, W. L. Kruer, M. Tabak, and A. B. Langdon, "Absorption of ultra-intense laser pulses," Phys. Rev. Lett. 69, 1383-1386 (1992).

3. J. Denavit, "Absorption of high-intensity subpicosecond lasers on solid density targets," Phys. Rev. Lett. 69, 3052 -3055 (1992).

4. G. Malka and J. L. Miquel, "Experimental confirmation of ponderomotive-force electrons produced by an ultrarelativistic laser pulse on a solid target," Phys. Rev. Lett. 77, 75-78 (1996).

5. M. Tabak, J. Hammer, M. E. Glinsky, W. L. Kruer, S. C. Wilks, J. Woodworth, E. M. Campbell, and M. D. Perry, "Ignition and high gain with ultrapowerful lasers," Phys. Plasmas 1, 1626-1634 (1994).

6. P. A. Norreys, M. Santala, E. Clark, M. Zepf, I. Watts, F. N. Beg, K. Krushelnick, M. Tatarakis, A. E. Dangor, $\mathrm{X}$. Fang, et al., "Observation of a highly directional $\mathrm{\gamma}$ -ray beam from ultrashort, ultraintense laser pulse interactions with solids," Phys. Plasmas 6, 2150-2156 (1999).

7. T. E. Cowan, J. Fuchs, H. Ruhl, A. Kemp, P. Audebert, M. Roth, R. Stephens, I. Barton, A. Blazevic, E. Brambrink, et al., "Ultralow emittance, multi-MeV proton beams from a laser virtual-cathode plasma accelerator," Phys. Rev. Lett. 92, 204801-204804 (2004). 
8. D. R. Farley, K. G. Estabrook, S. G. Glendinning, S. H. Glenzer, B. A. Remington, K. Shigemori, J. M. Stone, R. J. Wallace, G. B. Zimmerman, and J. A. Harte, "Radiative jet experiments of astrophysical interest using intense lasers," Phys. Rev. Lett. 83, 1982-1985 (1982).

9. E. Fourkal, J. S. Li, W. Xiong, A. Nahum, and C. M. Ma, "Intensity modulated radiation therapy using laseraccelerated protons: a monte carlo dosimetric study," Phys. Med. Biol. 48, 3977-4000 (2003).

10. E. L. Clark, K. Krushelnick, J. R. Davies, M. Zepf, M. Tatarakis, F. N. Beg, A. Machacek, P. A. Norreys, M. I. K. Santala, I. Watts, et al., "Measurements of energetic proton transport through magnetized plasma from intense laser interactions with solids," Phys. Rev. Lett. 84, 670673 (2000)

11. A. Maksimchuk, S. Gu, K. Flippo, and D. Umstadter, "Forward ion acceleration in thin films driven by a highintensity laser," Phys. Rev. Lett. 84, 4108-4111 (2000).

12. R. A. Snavely, M. H. Key, S. P. Hatchett, T. E. Cowan, M. Roth, T. W. Phillips, M. A. Stoyer, E. A. Henry, T. C. Sangster, M. S. Singh, et al., "Intense high-energy proton beams from petawatt-laser irradiation of solids," Phys. Rev. Lett. 85, 2945-2948 (2000).

13. Y. Sentoku, T. V. Liseikina, T. Z. Esirkepov, F. Califano, N. M. Naumova, Y. Ueshima, V. A. Vshivkov, Y. Kato, K. Mima, K. Nishihara, et al., "High density collimated beams of relativistic ions produced by petawatt laser pulses in plasmas,” Phys. Rev. E 62, 7271-7281 (2000).

14. A. Pukhov, "Three-dimensional simulations of ion acceleration from a foil irradiated by a short-pulse laser," Phys. Rev. Lett. 86, 3562-3565 (2001).

15. A. J. Mackinnon, M. Borghesi, S. Hatchett, M. H. Key, P. K. Patel, H. Campbell, A. Schiavi, R. Snavely, S. C. Wilks, and O. Willi, "Effect of plasma scale length on multi-MeV proton production by intense laser pulses," Phys. Rev. Lett. 86, 1769-1772 (2001).

16. K. Matsukado, T. Esirkepov, K. Kinoshita, H. Daido, T. Utsumi, Z. Li, A. Fukumi, Y. Hayashi, S. Orimo, M. Nishiuchi, et al., "Energetic protons from a few-micron metallic foil evaporated by an intense laser pulse," Phys. Rev. Lett. 91, 215001-215004 (2003).

17. Y. Sentoku, T. E. Cowan, A. Kemp, and H. Ruhl, "High energy proton acceleration in interaction of short laser pulse with dense plasma target," Phys. Plasmas 10, 20092015 (2003).

18. H. Habara, R. Kodama, Y. Sentoku, N. Izumi, Y. Kitagawa, K. A. Tanaka, K. Mima, and T. Yamanaka, "Fast ion acceleration in ultraintense laser interactions with an overdense plasma," Phys. Rev. E 69, 036407 -036416 (2004).
19. E. d'Humieres, E. Lefebvre, L. Gremillet, and V. Malka, "Proton acceleration mechanisms in high-intensity laser interaction with thin foils," Phys. Plasmas 12, 062704062715 (2005).

20. L. O. Silva, M. Marti, J. R. Davies, R. A. Fonseca, C. Ren, F. S. Tsung, and W. B. Mori, "Proton shock acceleration in laser-plasma interactions," Phys. Rev. Lett. 92, 015002-015005 (2004).

21. H. J. Lee, K. H. Pae, H. Suk, and S. J. Hahn, "Enhancement of high-energy ion generation by preplasmas in the interaction of an intense laser pulse with overdense plasmas," Phys. Plasmas 11, 1726-1729, (2004).

22. J. T. Seo, S. H. Yoo, and S. J. Hahn, "Effects of underdense preplasma on the energetic proton generation in ultraintense short laser pulse interaction with an overdense plasma slab,” J. Phys. Soc. Jpn. 76, 114501-114505 (2007).

23. T. Esirkepov, M. Yamagiwa, and T. Tajima, "Laser ion-acceleration scaling laws seen in multiparametric particle-in-cell simulations," Phys. Rev. Lett. 96, 105001 -105004 (2006).

24. H. Schwoerer, S. Pfotenhauer, O. Jackel, K. U. Amthor, B. Liesfeld, W. Ziegler, R. Sauerbrey, K. W. D. Ledingham, and T. Esirkepov, "Laser-plasma acceleration of quasimonoenergetic protons from microstructured targets," Nature 439, 445-449 (2006).

25. T. Nakamura, H. Sakagami, T. Johzaki, H. Nagatomo, K. Mima, and J. Koga, "Optimization of cone target geometry for fast ignition,” Phys. Plasmas 14, 103105103111 (2007).

26. J. T. Seo, S. H. Yoo, and S. J. Hahn, "High-quality energetic proton beams from an intense laser-plasma interaction with a micro-structured slab target," J. Kor. Phys. Soc. 53, 3229-3235 (2008).

27. J. P. Verboncoeur, A. B. Langdon, and N. T. Gladd, "An object-oriented electromagnetic PIC code," Comp. Phys. Comm. 87, 199-211 (1995).

28. T. Okada, A. A. Andreev, Y. Mikado, and K. Okubo, "Energetic proton acceleration and bunch generation by ultraintense laser pulses on the surface of thin plasma targets," Phys. Rev. E 74, 026401-026405 (2006).

29. B. M. Hegelich, B. J. Albright, J. Cobble, K. Flippo, S. Letzring, M. Paffett, H. Ruhl, J. Schreiber, R. K. Schulze, and J. C. Fernandez, "Laser acceleration of quasimonoenergetic $\mathrm{MeV}$ ion beams,” Nature 439, 441-444 (2006).

30. M. Chen, Z.-M. Sheng, Q.-L. Dong, M.-Q. He, Y.-T. Li, M. A. Bari, and J. Zhang, "Collisionless electrostatic shock generation and ion acceleration by ultraintense laser pulses in overdense plasmas," Phys. Plasmas 14, 053102-053110 (2007). 